\title{
Diffusion induced phase separation with crystallizable nylons. I. Mass transfer processes for nylon 4,6
}

\author{
A.M.W. Bulte, M.H.V. Mulder, C.A. Smolders, H. Strathmann \\ Faculty of Chemical Technology, University of Twente, P.O. Box 217, 7500 AE Enschede, The Netherlands
}

Received 26 July 1995; revised 21 May 1996; accepted 21 May 1996

\begin{abstract}
Mass transfer during membrane formation by means of phase inversion for a polymeric system with both a solid-liquid and a liquid-liquid equilibrium was studied on the basis of the theory developed by Reuvers and Smolders. During the first moments of immersion in the coagulation bath, the concentrations at the interface between bath and film are governed by the virtual liquid-liquid equilibrium. This equilibrium no longer exists at a larger time scale. The interfacial concentrations as a result of the local liquid-liquid equilibrium during mass transfer are located deeply in the crystallization region or solid-liquid demixing area and after an induction time the solid-liquid phase separation (crystallization) takes place when membranes are formed with an initial polymer concentration of $20 \%$ or larger. The calculated initial concentration profiles show a shallow pattern in polymer content for the films with initial concentration of 20 and $25 \%$. From the calculated initial concentration profiles an isotropic morphology in the final membrane can be expected. A steep increase of the polymer concentration at the interface was observed for the more concentrated films correlated with a skinned morphology in the final membrane.
\end{abstract}

Keywords: Membrane formation; Mass transfer; Crystallization; Spherulitic and axialitic morphology

\section{Introduction}

Membranes can be prepared by various techniques of which immersion precipitation is widely used [1]. In this process a polymer solution is cast as a thin film and subsequently immersed in a nonsolvent bath, thereby inducing diffusional exchange of solvent and non-solvent, resulting in phase separation. With semi-crystalline polymers, such as aliphatic nylons, two types of phase separation processes may occur. Liquid-liquid demixing resulting in a porous structure with pores that are formed from the nucleated polymer lean phase and solid-liquid demixing with crystalline parts in the membrane. The pores in this case are the voids in between the partly crystalline units.

It has been found that for the ternary system nylon 4,6, formic acid and water crystallization or solid-liquid demixing occurs, resulting in a spherulitic or axialitic morphology of the membrane when the casting solution has a rather high polymer concentration [2]. At lower polymer concentrations the typical cellular morphology, which is a result of liquid-liquid demixing, has been observed. The equilibrium thermodynamics of this system has been described previously [3]. Thermodynamically, crys- 


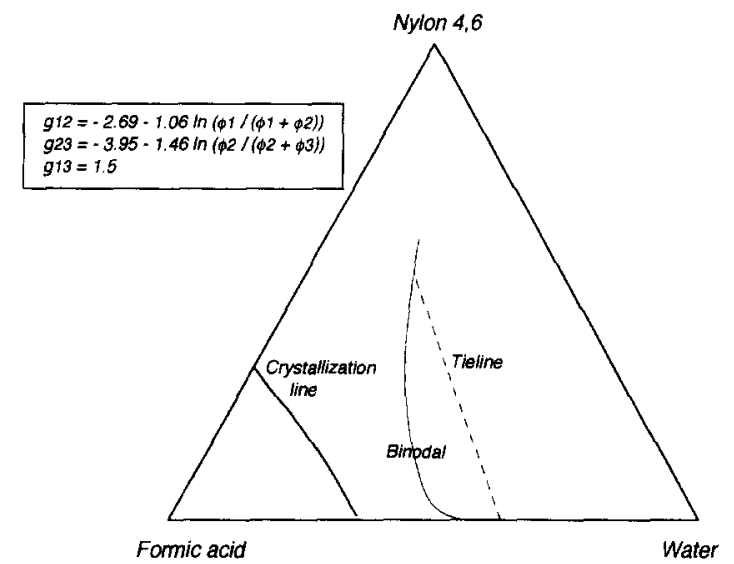

Fig. 1. Isothermal (phase) diagram at $30^{\circ} \mathrm{C}$ for the ternary system water (1), formic acid (2) and nylon 4,6 (3), with the experimentally determined crystallization line and the calculated liquidliquid demixing gap depicted [3]. The virtual binodal does not represent existing equilibria in this thermodynamic phase diagram. Its location, however, is important for membrane formation.

tallization is the favorable phase inversion process. The phase diagram with the parameters used is given in Fig. 1.

Knowledge about the thermodynamics of the membrane forming system is a first step towards understanding the formation of phase inversion membranes. It has been shown, however, that kinetic conditions may result into another phase separation process than predicted by the equilibrium thermodynamic properties of the system [2,3]. In order to understand the mass transfer of the components during the diffusion processes Reuvers and Smolders [4] have developed a model on the basis of earlier work of Cohen and co-workers [5]. Since then a number of papers have been published on mass transfer during a diffusion induced phase separation process [6-8]. Two different kinds of circumstances leading to different types of morphology in the final membranes, in both cases determined by liquid-liquid demixing [9], have been found. Firstly, delayed onset of demixing which results in membranes with a dense skin and a porous sub-layer with isolated pores. The second type, instantaneous demixing, being visualized by the occurrence of immediate turbidity in the film after immersion, results in membranes with a porous skin. The model developed by Reuvers and Smolders [9] allows predictions concerning the type of membrane morphology that can be expected.
In order to develop a further insight concerning the morphology of a membrane, determined by solid-liquid demixing, mass transfer in ternary systems should be studied, while including the crystallization process. Many polymers, such as aliphatic nylons, poly(phenylene oxide) or cellulose acetate, are able to crystallize. While it is known that this influences the properties of the resulting membranes, the crystallization phenomenon has always been neglected in the models describing mass transfer during immersion precipitation, although Koenhen and Smolders [10] and Altena and Smolders [11] have studied the thermodynamics of crystallization in binary and ternary systems for poly(phenylene oxide) and cellulose acetate respectively.

The objective for this paper is to develop a better understanding of the initial concentration profiles of the different components during phase inversion in which crystallization is involved, and to find a relationship between the concentration profiles and the final morphology in the membranes.

\section{Theory}

\subsection{Mass transfer model}

Immersion precipitation is governed by a solvent and a non-solvent flux perpendicular to the film surface as may be deduced from Figs. 2 and 3. The final morphology of a membrane is largely influenced by the concentration profile in the film at the moment that the phase separation processes start.

Before immersion of the polymer film into the coagulation bath the concentration of polymer is homogeneously distributed in the film and no concentration gradients exist in the coagulation bath. After immersion the in-flow of non-solvent (component 1) into the film and out-flow of solvent (component 2) effects the concentration of all three components in the film. The polymer does not dissolve in the coagulation bath; component (3) stays in the film. Because of a higher flux of solvent $\left(J_{2}\right)$ relative to the flux of non-solvent $\left(J_{1}\right)$, the polymer concentration in the film generally increases as is indicated schematically in Fig. 2. The concentration gradients are generally steeper closer to the interface. Mass transfer can only be described if for both the 


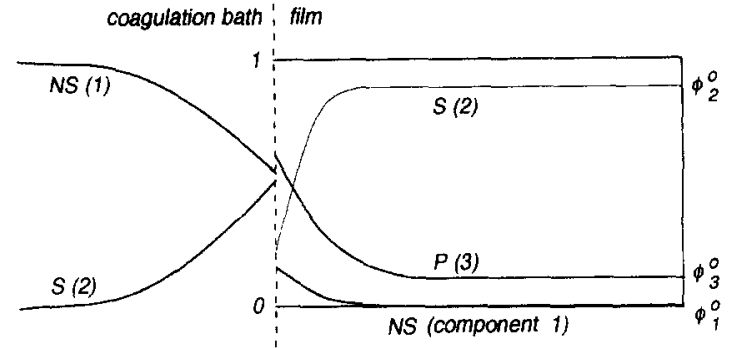

Fig. 2. Schematic concentration profiles of the three components in the film, polymer (3) with an initial concentration of $\phi_{3}^{0}$, solvent (2) with an initial concentration of $\phi_{2}^{0}$ and non-solvent (1) with the initial non-solvent concentration $\phi_{1}^{0}$ equal to zero in this case, and of solvent (2) and non-solvent (1) in the coagulation bath.

bath and the film differential flux equations are derived. The approach as developed by Reuvers et al. [4] will be followed here.

\subsubsection{The diffusional equations for the film}

Since in most cases $\left|J_{1}\right|<\left|J_{2}\right|$ the film shrinks and therefore the position of the interface is gradually changing in time. Mass transfer must be described with a moving boundary model. The mass transfer is based on a phenomenological model in which a flux is related to thermodynamic driving forces.

$J_{i}^{c}=\sum_{j=1}^{2} L_{i j} X_{j}$

with

$X_{j}=-\frac{\delta \mu_{j}}{\delta x}$ for which $\mu_{j}$ is the chemical potential of component $j$ per $\mathrm{kg} j$ and $x$ is the cartesian spatial coordinate perpendicular to the membrane surface, and the $L_{i j}$ 's are the phenomenological coefficients. $J_{i}^{c}$ gives the mass flux in $\mathrm{kg} / \mathrm{m}^{2} \mathrm{~s}$. According to Crank [12] a moving boundary problem can be treated by defining the fluxes relative to a polymer fixed frame of reference, since the polymer stays behind in the film, and acts as a reference component.

The fluxes are defined as follows:

$J_{i}^{c}=c_{i}\left(\bar{\nu}_{i}-\bar{\nu}_{3}\right)$

Where $\bar{\nu}_{i}$ and $\bar{\nu}_{3}$ are the average velocities of component $i$ and 3 relative to the lab fixed frame of reference and $c_{i}$ is the concentration of component $i$ in $\mathrm{kg} / \mathrm{m}^{3}$.

For treating the moving boundary problem the coordinate $m$ is introduced,

$m(x, t)=\int_{0}^{x} \phi_{3}(x, t) \mathrm{d} x$

The continuity equation for this system is given as:

$$
\frac{\delta \phi_{i}(x, t)}{\delta t}=\frac{\delta \tilde{J}_{i}(x, t)}{\delta x}
$$

The tilde $\sim$ denotes that $\tilde{J}_{i}$ is a flux relative to the laboratory fixed frame of reference.

Eq. (4) can be restated according to Eq. (5).

$\tilde{J}_{i}=\phi_{i} \bar{\nu}_{i}\left(=c_{i} \eta_{i} \bar{\nu}_{i}\right)$

The flux, $\tilde{J}_{i}$, equals the volume fraction of component $i, \phi_{i}$, multiplied with its velocity, $\bar{\nu}_{i}$. The

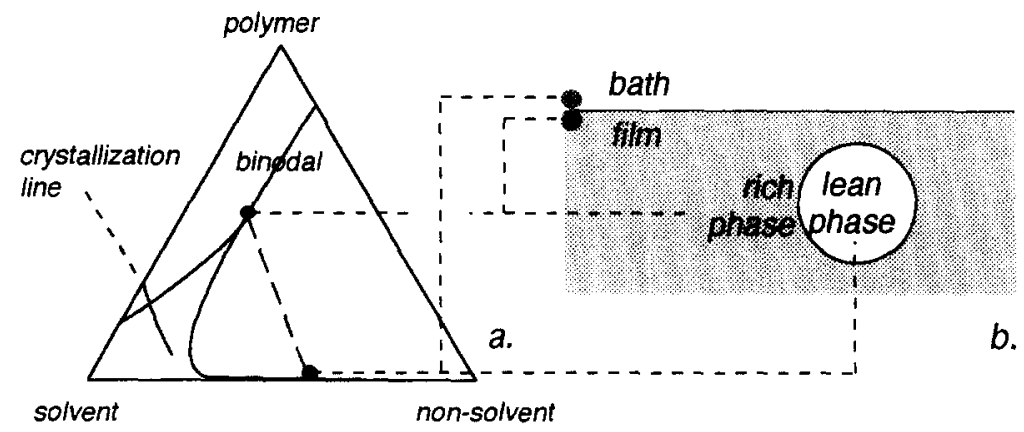

Fig. 3. (a) Conditions for local equilibrium at the interface film-coagulation bath and in the nucleus of the polymer lean phase. (b) Part of a film contacted with an immersion bath. Liquid-liquid phase separation has taken place. The concentrations of the polymer rich and polymer lean phase and also at the film and bath side of the film-bath interface are based on the same equilibrium. 
volume fraction can be expressed in the concentration times the partial specific volume, $\eta_{i}$.

It is assumed that the partial specific volumes, $\eta_{i}$, are constant and equal to the specific volume. The volume fixed and the laboratory fixed frames of reference are related to each other by a constant factor. The fluxes $\tilde{J}_{i}$ (lab fixed frame of reference) and $J_{i}$ (polymer fixed frame of reference) are related as follows:

$J_{i}=\tilde{J}_{i}-\left(\phi_{i} / \phi_{3}\right) \tilde{J}_{3}$

By using the transformation (3) and the continuity equation, Eq. (1) is transformed to Eq. (8).

$J_{i}=-\sum_{j=1}^{2} \eta_{i} \phi_{3} L_{i j} \frac{\delta \mu_{i}}{\delta m} \quad i=1,2$

The relation between the compositions at the directional coordinate $m$ and time $t$ is given by Eq. (8) with a concentration dependent phenomenological coefficient $L_{i j}$.

$$
\frac{\delta \phi_{i} / \phi_{3}}{\delta t}=\frac{\delta}{\delta m} \sum_{j=1}^{2} \eta_{i} \phi_{3} L_{i j} \frac{\delta \mu_{i}}{\delta m} \quad i=1,2
$$

The fluxes are calculated by integration of the left hand term of Eq. (8).

\subsection{The diffusional equations for the coagulation bath}

The binary diffusion processes in the coagulation bath can be described by Fick's second law.

$$
\frac{\delta \phi_{i}}{\delta t}=\frac{\delta}{\delta x}\left\{\mathbb{D} \phi_{i} \frac{\delta \phi_{i}}{\delta x}\right\}
$$

For the bath the position coordinate $y$ is used as is given in Fig. 3.

$y=-x+X(t)$

Transferring Eq. (9) with regard to the moving frame gives the differential equations for the bath.

As can be understood easily the change in the interface position $(\mathrm{d} X(t) / \mathrm{d} t)$ relative to the laboratory frame of reference is reflected by the sum of the volume fluxes $J_{1}$ and $J_{2}$ at $y=0$, with $J_{1}$ being negative relative to the $y$ coordinate. The sum of the fluxes has a positive value in most cases, which implies that the coagulation bath expands and the film shrinks.

$$
\begin{aligned}
& \frac{\delta \phi_{i}}{\delta t}=\frac{\delta}{\delta y}\left\{D\left(\phi_{i}\right) \frac{\delta \phi_{i}}{\delta y}\right\}-\frac{\delta \phi_{i}}{\delta y} \frac{\delta X(t)}{\delta t} \\
& \frac{\delta X(t)}{\delta t}=J_{1}(y=0)+J_{2}(y=0)
\end{aligned}
$$

\subsection{Crystallization in relation to mass transfer}

In the previous section the mass transfer model as developed by Reuvers et al. [4] has been explained. This mode! only deals with one phase separation process, defined by the liquid-liquid demixing boundary, and discusses the mass transfer processes before the actual phase separation process has started. For the membrane forming system discussed in this study two types of phase equilibria are involved: liquid-liquid and solid-liquid demixing boundaries, i.e. the binodal curve and the crystallization line respectively. In this section the process of crystallization will be discussed in relation to mass transfer for this ternary system.

Crystallization always needs some degree of supersaturation, i.e. it does not set in at the moment the equilibrium solid-liquid line in the phase diagram is crossed. First a nucleus with a critical size must be created (thermodynamic reasons) and the crystallization process requires an induction time. The creation of the nuclei or embryos requires the polymer molecules to rearrange in an ordered structure, the crystalline lattice. Examples of schematic drawings of such ordered structures are given in Ref. [13], with the polymer molecules folded back upon themselves. It has been shown that polymers crystallize in spherulites or axialites [14]. For the same considerations as described by Khoury and Passaglia [15] we do not adopt the fringed micelle model. In order to create ordered crystalline structures two steps are involved. Firstly the polymer coil must move to the growing polymer surface and secondly the polymer molecule must fold itself in a specific order in the crystalline lattice, the surface reaction. These kinetic reasons also make clear that crystallization or solidliquid phase separation needs an induction time. 
At the very first moment of immersion of the film in the coagulation bath the crystallization process has not started yet. We assume that from a very small time interval after immersion of the film continuous chemical potential gradients are present (i.e. local equilibrium is assumed) [16]. In case of systems in which a liquid-liquid demixing gap is present the conditions at the interface are clear. A local interfacial equilibrium between the film and the coagulation bath is a liquid-liquid equilibrium that is given by a pair of points on the binodal connected by a tie-line. The composition on the film side is given by a polymer rich phase, the composition in the bath at the interface is the corresponding lean phase (see Fig. 3).

For systems with crystallizable polymers the situation is more complex. At the first moments of immersion two liquid phases are contacted while in later stages (two or three seconds or longer) solidliquid phase separation takes place. The first moments after immersion cannot be dictated by a solid-liquid demixing border, since the local equilibria at the interface are concerned with a liquid-liquid interface, bath-film. It has been shown that a binodal curve does exist for the system nylon 4,6, formic acid and water [2], because at the lower concentrations a foam-like morphology has been obtained. This is a clear indication that a liquid-liquid demixing gap is present for this ternary system [2]. It can be assumed that in the first moments after immersion a local liquid-liquid phase equilibrium is present at the interface. The exact composition at both sides of the interface are given by the nodes of a tie-line with the binodal.

These first moments of immersion can be described by the model of Smolders and Reuvers. The parameters for the model are summarized below.

\subsection{The initial and boundary conditions}

The following initial conditions are valid [4]: at $t=0$ the compositions of the film and of the coagulation bath are completely homogeneous. The film consists of the original polymer solution and nonsolvent eventually added to the solution. The bath has the initial concentration for non-solvent (and eventually added solvent). The indices $b$ and $f$ stand for the coagulation bath and the film, respectively, $M$ gives the site near the glass plate.

$$
\begin{array}{lll}
0 \leq m \leq M: & \phi_{i}^{\mathrm{f}}(m, 0)=\phi_{i}^{\mathrm{f}} & i=1,2,3 \\
y>0: & \phi_{i}^{\mathrm{b}}(y, 0)=\phi_{i}^{\mathrm{b}} & i=1,2
\end{array}
$$

These equations are valid for $t=0$; they state that both in the film and in the bath no concentration gradients are present just before immersion of the film in the bath.

At time $t>0$ after the very first moments of immersion local equilibrium is assumed leading to the boundary conditions at $m=0$ and $y=0$ :

$\mu_{i}^{\mathrm{f}}(m=0, t)=\mu_{i}^{\mathrm{b}}(y=0, t) \quad i=1,2,3$

There is no accumulation of one of the components at the interface. Components that diffuse out of the film, diffuse into the coagulation bath and vice versa. At the interface the following condition is valid:

$J_{i}(m=0, t)=-J_{i}(y=0, t) \quad i=1,2$

\subsection{Calculation procedure and assumptions}

For description of the chemical potentials in the ternary system the Flory-Huggins theory is used [3].

The calculation procedure as described by Reuvers and Smolders [9] is used.

1. For the boundary conditions at the interface a tie-line is chosen on the binodal curve.

2. Solvent and non-solvent fluxes are calculated for the film. Concentration gradients are calculated with Eq. (8).

3. The value for the sum of the fluxes is used to calculate the non-solvent flux from the coagulation bath. Concentration profiles are calculated for the coagulation bath by means of Eq. (11).

4. The procedures 1,2 and 3 are repeated until the flux of non-solvent is equal for both the film and the bath by adjusting the interfacial concentration.

The last step before the concentration profiles for our system can be calculated is the determination of the phenomenological coefficients for the film part and the diffusioncoefficient for the calculation of the concentrations in the film.

The same assumptions as have been described by Reuvers and Smolders [4,9] have been made, in 
which the phenomenological coefficients $L_{i j}$ are related to the Maxwell-Stefan coefficients $R_{i j}$.

According to Reuvers the following relation is derived:

$$
\begin{aligned}
L_{i j} & =\left|\begin{array}{ll}
L_{11} & L_{12} \\
L_{21} & L_{22}
\end{array}\right| \\
& =\left[\begin{array}{cc}
\frac{c_{1}\left(c_{1} R_{12}+c_{3} R_{23}\right)}{\alpha} & \frac{c_{1} c_{2} R_{12}}{\alpha} \\
\frac{c_{1} c_{2} R_{12}}{\alpha} & \frac{c_{2}\left(c_{2} R_{12}+c_{3} R_{13}\right)}{\alpha}
\end{array}\right]
\end{aligned}
$$

with

$$
\alpha=c_{3}\left(c_{2} R_{12} R_{23}+c_{1} R_{12} R_{13}+c_{3} R_{13} R_{23}\right)
$$

The Maxwell-Stefan parameters, $R_{12}, R_{13}$ and $R_{23}$ are binary parameters and can be derived from published binary data or experimentally determined. Furthermore it is assumed that $R_{12}=R_{21}$ (nonsolvent-solvent) according to the reciprocity relation. This value is taken as constant and can be related to the binary diffusion coefficient at infinite dilution, $\mathbb{D}\left(\phi_{1}=1\right)$. The coefficients $R_{13}$ and $R_{23}$ are concentration dependent. Friction between polymer and solvent can be related to a sedimentation coefficient, $s_{3}\left(\phi_{3}\right)$. The polymer-non-solvent parameter, $R_{13}$, is assumed to be linearly related to $R_{23}$.

$$
\begin{aligned}
& R_{12}\left(\phi_{1}\right)=R_{12}\left(\phi_{1}=1\right)=\frac{\eta_{1} R T}{M_{2} D\left(\phi_{1}=1\right)} \\
& R_{23}\left(\phi_{3}\right)=\frac{\left(1-\phi_{3}\right) \eta_{3}\left(\rho \eta_{2}-1\right)}{\phi_{3}} \frac{1}{s_{3}\left(\phi_{3}\right)} \\
& R_{13}=\left(\eta_{1} / \eta_{2}\right) R_{23}
\end{aligned}
$$

with $\eta_{i}$ and $\rho$ indicating the partial specific volume of component $i$ and the density, respectively.

The model system in this case is:

(1) non-solvent: water

(2) solvent: formic acid

(3) membrane forming polymer: nylon 4,6 .

The diffusion coefficient of a trace of formic acid in water $\left(\mathbb{D}\left(\phi_{1}=1\right)\right)$ has been measured with a Taylor capillary [18] and a value of $1.51 \times 10^{-9}$ $\mathrm{m}^{2} / \mathrm{s}$ at $30^{\circ} \mathrm{C}$ was found. With Eq. (16) the friction coefficient of non-solvent-solvent can then be calculated. Since the values for the density of solvent and

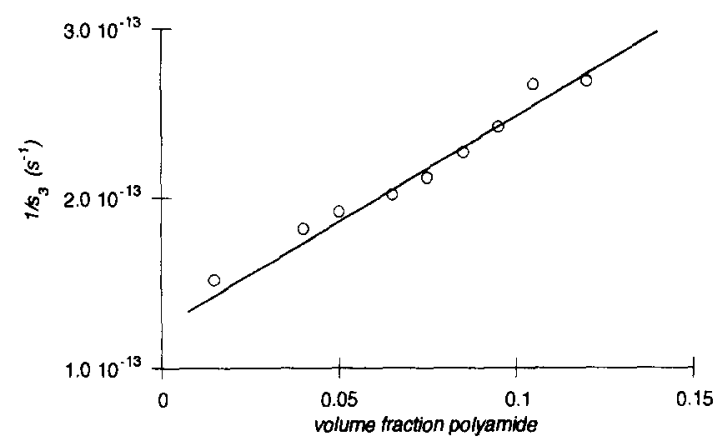

Fig. 4. Concentration dependence of $l / s_{3}$ (the sedimentation coefficient) that is linearly related to the coefficient $R_{23}$ for nylon 4,6 in formic acid as it is estimated from literature data.

polymer lie close to each other a sedimentation or flotation coefficient can not be experimentally determined. Data for sedimentation coefficients of various polyamides in different solvents are listed in the Polymer Handbook [19]. The order of magnitude of the coefficients is similar for all types of polyamides. The same concentration dependency is assumed as is found by Reuvers and Smolders [9] in which the sedimentation coefficients at infinite dilution is taken for an aromatic polyamide with a molecular weight of $25000 \mathrm{~g} / \mathrm{mol}$ in dimethylformamide with $3 \%$ of $\mathrm{LiCl}$ [19]. The sedimentation coefficient of nylon 4,6 in formic acid is estimated in this way. The inverse value of the sedimentation coefficient $\left(1 / s_{3}\right)$, which is linearly related to $R_{23}$, has been plotted in Fig. 4 . [17].

In the calculations the following relations are used

$F_{3}=0.638 \times 10^{\left(-8-2.97 \times \phi_{3}\right)}$

with

$R_{23}\left(\phi_{3}\right)=\frac{\eta_{3} R T}{M_{2} F_{3}}$

with the coefficient $F_{3}$ inversely proportional to $R_{23}, M_{2}$ the molecular weight of component 2 and $\eta_{3}$ the partial specific volume of the polymer.

The calculations are carried out during the first full second. The sensitivity of the calculations towards a change in the values for the friction coefficients will be evaluated.

\section{Results and discussion}

The calculated composition paths starting from various polymer concentrations are given in Fig. 5 . 


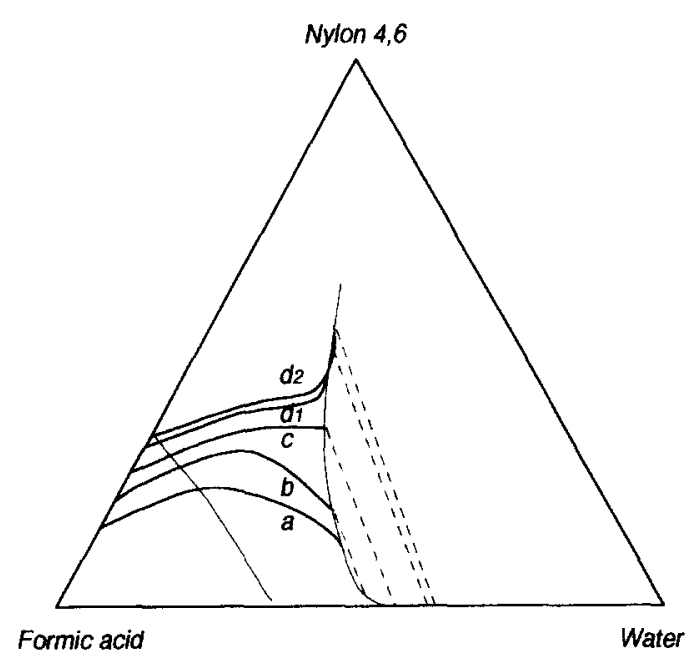

Fig. 5. Calculated composition paths for immersion of a film with initial polymer concentration of: (a) $15 \%$, (b) $20 \%$, (c) $25 \%$, (d $\left.{ }_{1}\right)$ $30 \%$ and $\left(\mathrm{d}_{2}\right) 32 \%$. The experimental crystallization line and the calculated binodal are given as determined in a previous paper [3].

A composition path as it has been defined by Reuvers gives information about the compositions at all positions; it is a curve representing all compositions existing in the polymer solution at one moment, or all compositions at all times at one position in the polymer solution assuming that the polymer solution and the coagulation bath are semi-infinite media under the time scale of consideration. The calculated profiles of the polymer concentration as a function of the position coordinate are given in Fig. 6.

The binodal curve (only valid when crystallization has not started yet) and the experimental crystallization line are given in Fig. 5. The composition path "a" (Fig. 5) can be considered as a delayed type of demixing in the sense of liquid-liquid demixing, which is consistent with the physical background of the system. The light transmission profiles showed a delay time for this case (see Fig. 7a). Crystallization in case " $a$ " does not take place prior to liquid-liquid phase separation; the membrane cast from a $15 \%$ polymer solution (see Fig. 8a.) shows a cellular morphology as a result of liquid-liquid demixing, even if some of the compositions are inside the crystallization region. The degree of supersaturation for crystallization is apparently not large enough to form nuclei of a critical size. The calculated compositions of curve " $a$ " are located in a region, in which samples used for determination of the equilibrium melting temperatures [3] showed crystallization only after several hours to several weeks. Crystallization from the melt generally requires at least a degree of undercooling of $30^{\circ} \mathrm{C}$ or more; the comparison with crystallization from the melt has further been worked out in [20].

The composition path " $b$ " is located deeper into the solid-liquid region (see Fig. 5). A delay time in the same order of magnitude as for case " $a$ ", 25 to $30 \mathrm{~s}$ (see Fig. 8b) can be found for the membrane formation with a polymer solution of $20 \%$. In this case the delay time is related to solid-liquid demixing, at least what is observed in the final morphology (Fig. 8b). It is very likely, however, that the first turbidity results from early nucleation and growth of a polymer lean phase (liquid-liquid demixing) followed by crystallization. Some remains of an early "cellular" morphology can be observed in the SEM photograph (Fig. 8b); the irregularly formed spherulites are connected with each other by ties, which are probably the remains of the "walls of cells".

Composition path "c"' (Fig. 5) has entered the crystallization region deeper compared to case " $b$ ". The profile as it is calculated according to Reuvers is "delayed" with respect to liquid-liquid demixing. The values for the delay time in case of membrane formation from a 25\% polymer solution (Fig. 7c) is 6 $\mathrm{s}$. Whether the first turbidity observed is a result of liquid-liquid or of solid-liquid phase separation cannot be concluded from the morphology determined by SEM (Fig. 8c), because remains of polymer lean nuclei, possibly resulting from liquid-liquid phase separation, have not been found. The delay time for solid-liquid demixing is smaller than the delay time for liquid-liquid demixing The larger degree of supersaturation with respect to crystallization has resulted in the formation of smaller spherulites compared to case " $b$ ".

Calculation of the concentration profiles during the first second as is described above is justified for the cases " $a$ ", " $b$ " and " $c$ ", because phase separation either by liquid-liquid or by solid-liquid demixing has not occurred yet.

Furthermore it can be concluded that a good correlation has been found between the calculated initial composition paths and the experimental re- 

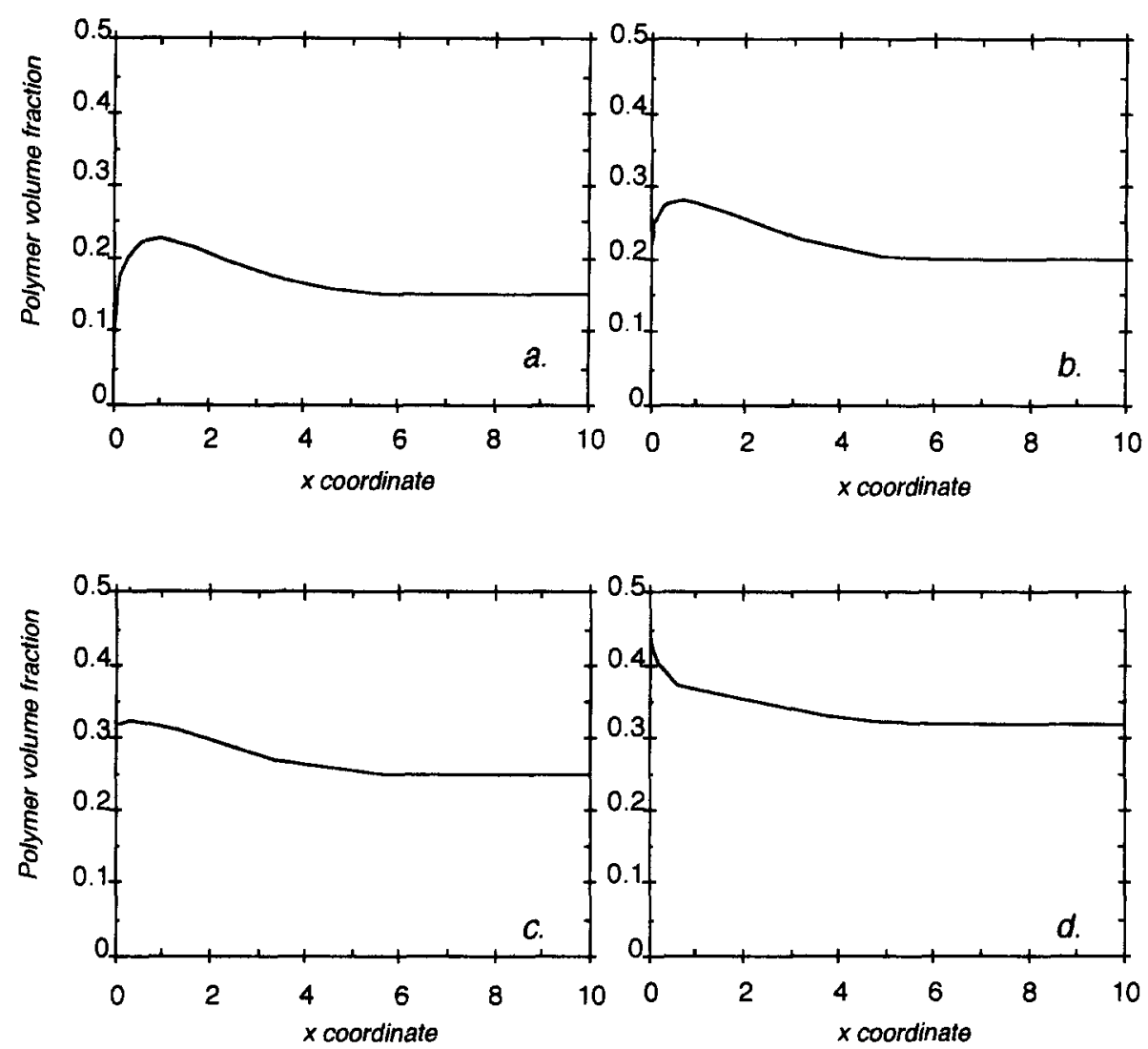

Fig. 6. (1) Concentration profiles for the polymer concentration versus the $x$ coordinate $(\mu \mathrm{m}$ ) valid for $t=1 \mathrm{~s}$ after immersion. The initial polymer concentration of the film is: (a) $15 \%$, (b) $20 \%$, (c) $25 \%$ and (d) $32 \%$. (2) Time normalized fluxes of solvent and non-solvent calculated during $t=1 \mathrm{~s}$.

sults. In all three cases mass transfer calculations do not predict a large interfacial polymer concentration. The structures of the three corresponding membranes, indeed, show a rather isotropic morphology (see Fig. 8).

The concentration profiles " $\mathrm{d}_{1}$ " and ' $\mathrm{d}_{2}$ " as calculated with the mass transfer model are considered as instantaneous with respect to liquid-liquid demixing, which might indicate that the first occurring demixing process is liquid-liquid phase separation. The calculated compositions inside the binodal area are just a result of the calculations, immediate phase separation after immersion into the bath will occur in the case of instantaneous liquid-liquid demixing. Whether instantaneous demixing with respect to liquid-liquid demixing, as it follows from the mass transfer model, takes place as a first phase separation process cannot be concluded from the experimentally obtained results. The immediate turbidity observed in the first second after immersion of the films (Fig. 7d) can be either due to the onset of liquid-liquid or solid-liquid phase separation. The driving force for crystallization, at the same time, is so large that a large number of nuclei can be created. An eventually formed polymer lean phase in a continuum of a polymer rich phase cannot be found after the crystallites have grown out to their maximum size. In the final morphology of the membrane the so formed crystalline structure dominates, and identification of the phase separation process responsible for the immediate turbidity after immersion is not important. Following the membrane formation visually two 


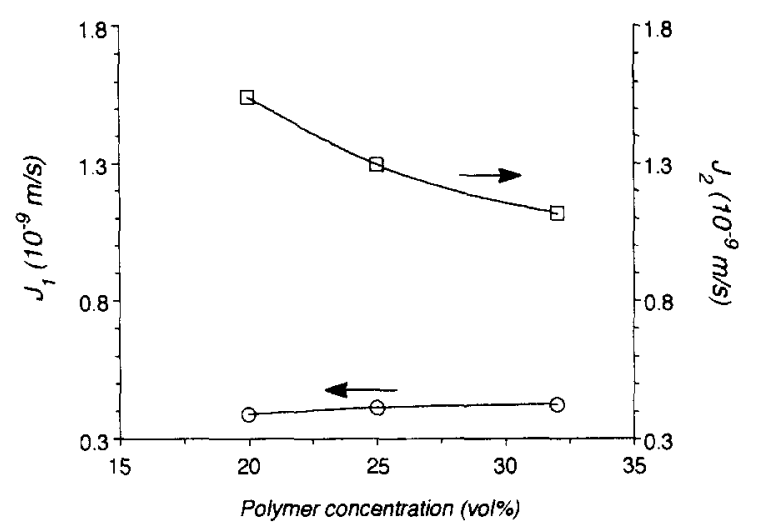

Fig. 6 (continued).

stages were observed: (1) The occurrence of an immediate haziness in the film after immersion, followed by (2) the creation of white spots after 2 to $3 \mathrm{~s}$ spreading out over the whole membrane area.

The large supersaturation in the cases " $\mathrm{d}_{1}$ " and " $\mathrm{d}_{2}$ " has resulted in an axialitic morphology in the final membranes because of a large nucleation den-
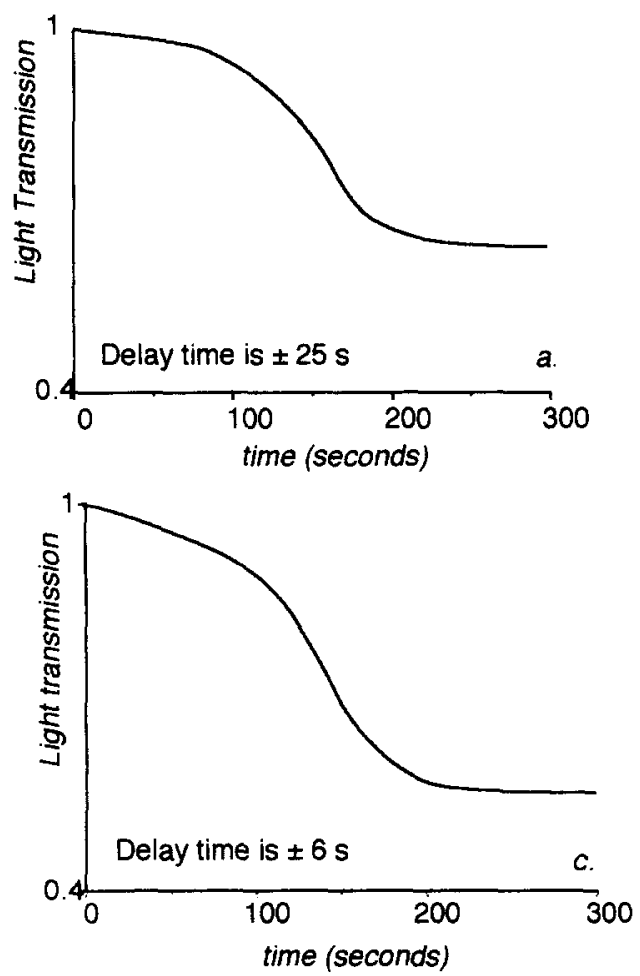

sity as has been described previously [2]. The large nucleation density is a result of a high nucleation frequency [20] in combination with moderate growth. It is not the result of a moderate nucleation frequency with slow growth that also leads to a high nucleation density.

The concentration profiles of the polymer $1 \mathrm{sec}$ ond after immersion of the film into the bath are given in Fig. 6(1) and the (time normalized) fluxes of solvent and non-solvent through the film-bath interface in Fig. 6(2). The solvent out-flux is the largest in case of a $15 \%$ solution and decreases as the polymer concentration in the initial solution is larger. For the $15 \%$ solution the out-flow of solvent is an order of magnitude larger that the inflow of non-solvent. In all cases the flux of solvent is larger than the non-solvent flux. The difference between these fluxes becomes smaller with increasing polymer concentration. As a consequence the non-solvent flux is the largest for the high polymer concentration despite the fact that the resistance for transport must be considerably larger in this case. The in-flux of
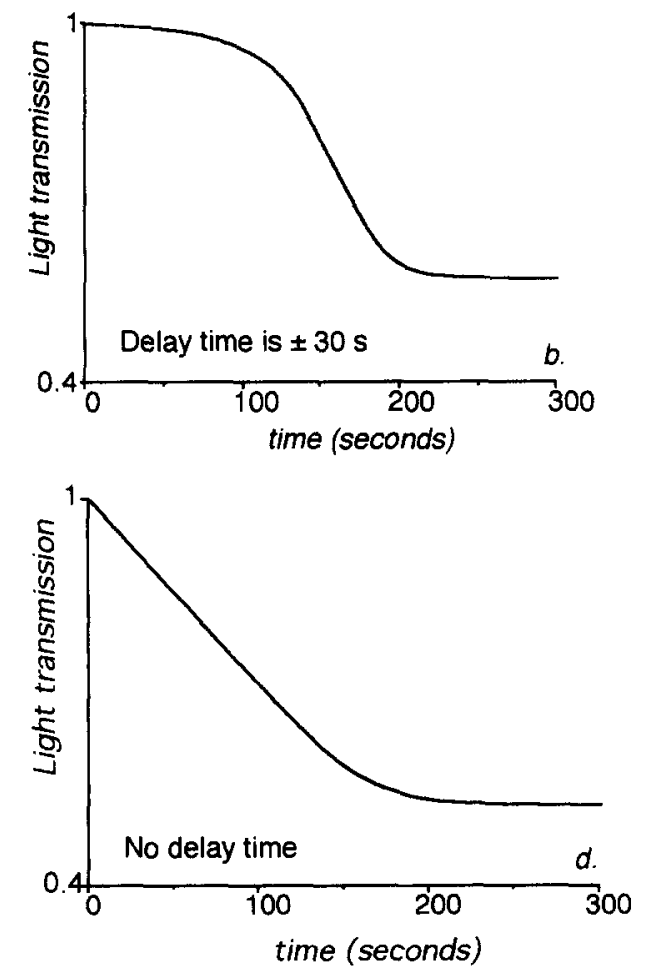

Fig. 7. Light transmission through the film as a function of time after immersion in a water bath for a film with initial polymer concentration of: (a) $15 \%$, (b) $20 \%$, (c) $25 \%$ and (d) $32 \%$. 

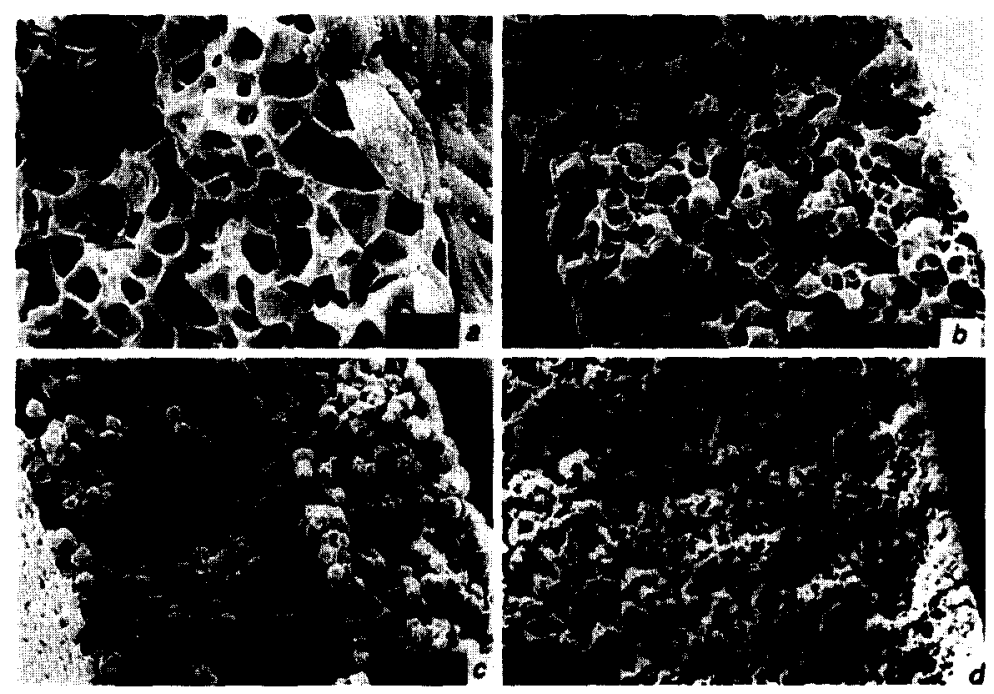

Fig. 8. Overview of the final morphology of the membranes cast with an initial polymer concentration of: (a) $15 \%$, (b) $20 \%$, (c) $25 \%$ and (d) $32 \%$ observed with scanning electron microscopy (SEM) [2].

non-solvent here is presumably hindered to a large extent by the "counter-current" flux of solvent, which is the largest for the lowest polymer concentration.

From Fig. 6 it follows that the polymer concentration at and close to the interface is in the cases " $a$ " and " $b$ " lower than the "bulk" polymer concentration. At the higher polymer concentrations ( $\geq 25 \%$ ) this effect does not take place. These results illustrate that a large solvent out-flux $\left(J_{2}\right)$ not necessarily implies that the interfacial polymer concentration increases compared to the initial polymer concentra- tion. The total compaction of polymer in the film as a result of a larger $J_{2}$ relative to $J_{1}$, which is related to $J_{2}+J_{1}$ with a negative $J_{2}$ relative to the film, is schematically given by the total area under the curve of a plot of the polymer volume fraction versus the $x$ coordinate (see Fig. 9a); area " 2 " represents a positive and area "1" a negative value.

Also for the cases " $a$ " and " $b$ " in Fig. 6(1) the area under the curves is positive, which is consistent with the calculated values for the solvent and nonsolvent flux through the interface. These calculations are in agreement with the morphology of the mem-
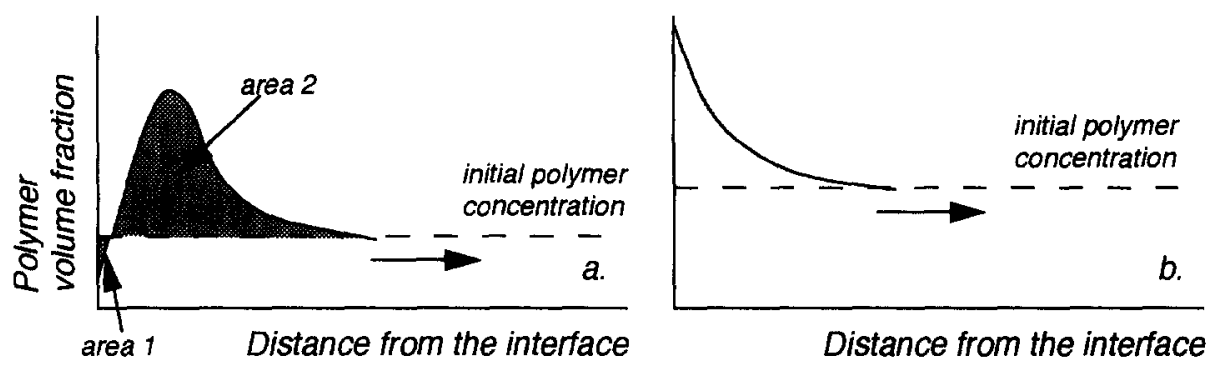

Fig. 9. Schematical polymer concentration as a function of the distance from the interface. In case " $a$ " the situation for lower interfacial polymer concentrations is drawn: " $b$ " reflects most cases in which the polymer concentration at the interface is higher than the initial polymer concentration. The area under the curve (area 2 - area 1) is a measure for the total compaction of polymer. 
branes observed in the corresponding Figs. 8a and 8b. A larger compaction in polymer concentration of the film at the interface that would lead to the formation of a skin is not observed.

For the higher polymer concentrations (curves $d_{1}$ and $\mathrm{d}_{2}$ ) the surface polymer concentration is much larger. Starting membrane formation with polymer concentrations of 20 ("b") and $25 \%$ ("c") of polymer in the casting solution, an isotropic morphology is observed. The morphology of the membranes cast from a 30 or $32 \%$ polymer solution show an asymmetric structure with a higher polymer concentration at the interface, which results in the formation of a skin. An instantaneously demixed film with respect to liquid-liquid phase separation usually gives ultrafiltration membranes with thin top-layers in the membrane with a thickness of $0.2 \mu \mathrm{m}$ or less. Here, however, ultrafiltration characteristics are not observed. The pore sizes are larger, typically in the microfiltration range [2], probably a result of crystallization. The polymer matrix shows an extra contraction as a result of crystallization, so the voids between the partly crystalline units, the pores, become larger. A detailed photograph of the top of a $32 \%$ membrane is given in Fig. 8d.

The results for the calculated composition paths do not significantly change if other Flory-Huggins interaction parameters are taken for the calculations. The uncertainty in the friction between polymer and solvent was evaluated by changing the "exponential pre-factor" of Eq. (19) with a factor of five. The exact location of the composition paths changes to some extent. The choice of a different parameter, however, does not lead to significantly different conclusions. The choice of the $R_{23}$ parameter gave consistent values for the calculated solvent flux (Fig. 6(2)) compared with the experimentally determined solvent fluxes by casting leaching experiments.

\section{Conclusions}

Mass transfer of the three components during membrane formation has been studied during the first moments of immersion of a nylon 4,6 solution in formic acid into the coagulation bath containing water. The results are related to the final state of the membrane morphology.
The types of mechanisms, delayed liquid-liquid demixing and solid-liquid demixing with an induction time, lead in most cases to a membrane with a morphology controlled by crystallization. The nucleation of crystalline material is highly dependent on the degree of supersaturation. Smaller spherulite size is a result of a larger nucleation density followed from a larger degree of supersaturation. If the supersaturation is too low crystallization of the polymer takes place after the cellular morphology was formed; nuclei of a crystalline phase could not be created on a short time scale. This type of morphology then is the result of liquid-liquid phase separation. Concentration profiles by solvent/non-solvent exchange develop more or less independently from the starting crystallization if the nucleation density is small. The flat composition paths (Fig. 5), which are calculated for 20 and $25 \%$ of initial polymer concentration in the film, give rise to an isotropic morphology in the final membrane.

Mass transfer calculations and SEM photographs showed that higher initial polymer concentrations, 30 or $32 \%$ polymer solutions, lead to membranes with higher interfacial polymer concentrations resulting in membranes with a (thin) skin. The higher degree of supersaturation resulted in an axialitic morphology.

It can be concluded that a further insight is developed in membrane formation in which crystallization is involved. The type of morphology, cellular, spherulitic or axialitic is mainly dependent on the degree of supersaturation that is a result of exchange of non-solvent and solvent. Calculation of the initial composition path is therefore an essential procedure for understanding and predicting the membrane morphology.

\section{List of symbols}

A surface $\left(\mathrm{m}^{2}\right)$

$c_{i} \quad$ concentration component $i\left(\mathrm{~kg} / \mathrm{m}^{3}\right)$

ID Fickian diffusion coefficient $\left(\mathrm{m}^{2} / \mathrm{s}\right)$

$F_{3} \quad$ coefficient $\left(\mathrm{m}^{2} / \mathrm{s}\right)$

$g_{i j} \quad$ Flory-Huggins interaction parameter between the components $i$ and $j$

$J_{i} \quad$ volume flux of component $i$ relative to the polymer volume frame of reference $(\mathrm{m} / \mathrm{s})$ 
$\tilde{J}_{i} \quad$ volume flux of component $i$ relative to the fixed frame of reference $(\mathrm{m} / \mathrm{s})$

$J_{i}^{c} \quad$ mass flux of component $i$ relative to the polymer volume frame of reference $\left(\mathrm{kg} / \mathrm{m}^{2} \mathrm{~s}\right)$

$L_{i j} \quad$ phenomenological coefficient for transport of $i$ in $j\left(\mathrm{~kg} \mathrm{~s} / \mathrm{m}^{3}\right)$

$m$ place volume coordinate for the polymer volume frame of reference

$M$ total volume of component 3

$M_{2}$ molecular weight of component $2(\mathrm{~kg} / \mathrm{mol})$

NS non-solvent

$\mathrm{P}$ polymer

$R \quad$ gas constant $(\mathrm{J} / \mathrm{mol} \mathrm{K})$

$R_{i j}$ Maxwell-Stefan coefficient between component $i$ and $j\left(\mathrm{~m}^{3} /(\mathrm{kg} \mathrm{s})\right)$

S solvent

$s_{3} \quad$ sedimentation coefficient (s)

$t$ time (s)

$T$ temperature (K)

$V$ volume $\left(\mathrm{m}^{3}\right)$

$\bar{v}_{i} \quad$ average velocity of component $i$ relative to the fixed frame of reference $(\mathrm{m} / \mathrm{s})$

$x$ coordinate in the fixed frame of reference in the film (m)

$y$ coordinate in the polymer volume frame of reference in the coagulation bath $(\mathrm{m})$

$\phi_{i} \quad$ volume fraction of component $i(-)$

$\mu_{i}$ chemical potential of component $i(\mathrm{~J} / \mathrm{kg}=$ $\mathrm{m}^{2} / \mathrm{s}^{2}$ )

$\eta_{i}$ partial specific volume of component $i$ $\left(\mathrm{m}^{3} / \mathrm{kg}\right)$

\subsection{Indices}

(1) non-solvent; water

(2) solvent; formic acid

(3) polymer; nylon 4,6

(b) coagulation bath

(f) film

\section{Acknowledgements}

The authors wish to thank the Computer Information Center of the University of Twente for help with the model calculation, Dr. Ir. R.M. Boom for explanation and handing over the computer programs and stimulating discussions and to DSM, The Netherlands for funding of this project.

\section{References}

[1] M.H.V. Mulder, Basic Principles of Membrane Technology, Kluwer, Dordrecht, 1991.

[2] A.M.W. Bulte, B. Folkers, M.H.V. Mulder and C.A. Smolders, Membranes of semi-crystalline aliphatic polyamide nyIon 4,6: formation by diffusion-induced phase separation, J. Appl. Polym. Sci., 50 (1993) 13.

[3] A.M.W. Bulte, E. Naafs, F. van Eeten, M.H.V. Mulder, C.A. Smolders and H. Strathmann, Equilibrium thermodynamics of the ternary membrane forming system nylon 4,6, formic acid and water, Polymer, 37 (1996) 1647.

[4] A.J. Reuvers, J.W.A. van den Berg and C.A. Smolders, Formation of membranes by means of immersion precipitation. Part I. A model to describe mass transfer during immersion precipitation, J. Membrane Sci., 34 (1987) 45.

[5] C. Cohen, G.B. Tanny and S. Prager, Diffusion-controlled formation of porous structures in ternary polymer solutions, J. Polym. Sci., Polym. Phys. Ed., 17 (1979) 477.

[6] C.S. Tsay, A.J. McHugh, Mass transfer modelling of asymmetric membrane formation by phase inversion, J. Polym. Sci., Polym. Phys. Ed., 28 (1990) 1327.

[7] Ph. Radovanovics, S.W. Thiel and S.-T. Hwang, Formation of asymmetric polysulfone membranes by immersion precipitation. I. Modelling mass transfer during gelation, J. Membrane Sci., 65 (1992) 213.

[8] K. Kimmerle, Quantitative Betrachtung de Phaseninversionsprozesses bei der Herstellung von Membranen, Ph.D. Thesis, University of Stuttgart, 1988.

[9] A.J. Reuvers and C.A. Smolders, Formation of membranes by means of immersion precipitation. Part II. The mechanism of formation of membranes prepared from the system CA/acetone/water, J. Membrane Sci., 34 (1987) 67.

[10] D.M. Koenhen and C.A. Smolders, Phase-separation phenomena in solutions of poly(2,6-dimethyl-1,4 phenylene oxide). I. Thermodynamic parameters of solutions in toluene, J. Polym. Sci., Polym. Phys. Ed., 15 (1977) 155.

[11] E.W. Altena and C.A. Smolders, Phase separation phenomena in solutions of cellulose acetate. I. Differential scanning calorimety of cellulose acetate in mixture of dioxane and water, J. Polym. Sci., Polym. Symp., 69 (1981) 1.

[12] J. Crank, The Mathematics of Diffusion, 2nd edn., Clarendon Press, Oxford, 1975.

[13] A. Keller, Philos. Mag., 2 (1957) 1171.

[14] H.D. Keith and F.J. Padden, Jr., A phenomenological theory of spherulitic crystallization, J. Appl. Phys., 34 (1963) 2409.

[15] F. Khoury and E. Passaglia, The morphology of crystalline synthetic polymers, in N.B. Hannay (Ed.), Treatise on Solid State Chemistry, Vol. 3, Crystalline and Non-Crystalline Solids, Plenum Press, NY, 1976, Chap. 6.

[16] S.R. De Groot and P. Mazur, Non-equilibrium Thermodynamics, North-Holland, Amsterdam, 1962. 
[17] R.M. Boom, Th. van den Boomgaard and C.A. Smolders, Mass transfer and thermodynamics during immersion precipitation for a four-component system with two polymers, J. Membrane Sci., 90 (1994) 231.

[18] E.D. Snijders, Metal Hydrides as Catalysts and Hydrogen Carriers, Ph.D. Thesis, University of Twente.

[19] J. Brandrup, E.H. Immergut, Polymer Handbook, 3rd edn., Wiley, NY, 1989.
[20] A.M.W. Bulte, B. Folkers, M.H.V. Mulder, C.A. Smolders and H. Strathmann, Diffusion induced phase separation with crystallizable nylons. II. Kinetic and thermodynamic parameters for membrane formation, Macromolecules, (1994) submitted. 